\title{
BENEFICIOS ECONÓMICOS DE LA CONSERVACIÓN DE LAS ÁREAS PROTEGIDAS ANDINAS
}

ECONOMIC BENEFITS OF CONSERVATION OF ANDEAN PROTECTED AREAS

\section{FABIÁN RODRÍGUEZ-ESPINOSA ${ }^{1,2}$, REMIGIO GALARRAGA. ${ }^{3}$, ROBERTO SALAZAR- CÓRDOVA $^{4}$, NIXON NARVÁEZ ${ }^{5}$, PAOLA ANANGANÓ-AYALA ${ }^{1}$}

'DEPARTAMENTO DE CIENCIAS DE LA TIERRA Y CONSTRUCCIÓN, UNIVERSIDAD DE LAS FUERZAS ARMADAS ESPE, Sangolquí, Ecuador. ffrodriguez3@espe.edu.ec; mpanangano@espe.edu. ec

${ }^{2}$ FACULTAD DE ECONOMÍA, PONTIFICIA UNIVERSIDAD CATÓLICA DEL ECUADOR, Quito, Ecuador.

${ }^{3}$ Gerente, DESARROLLO SUSTENTABLE AGUA Y SALUD CORIDEAS CIA. LTDA., Quito, Ecuador. remigala@gmail.com; corideas@gmail.com

${ }^{4}$ CEO \& Senior Researcher, HEXAGON GROUP/CSP - INC. TECH. LATAM\&UK, Santiago, Chile. rsalazar@hexagon-group.net.

${ }^{5}$ CENTRO DE GESTIÓN DEL CONOCIMIENTO EN SECRETARÍA DE AMBIENTE MUNICIPIO DE QUITO, Quito, Ecuador. nixonnarvaez@yahoo.es

Recibido: 01 de septiembre de 2018 / Aceptado: 22 de diciembre de 2018

\section{RESUMEN}

Sobre la base de la formación una mancomunidad cuyo objetivo principal es cuidar el agua de los páramos a través de corredores ecológicos se realizó una revisión y actualización de un estudio previo de valoración económica de los beneficios de los bienes y servicios del Sistema Nacional de Áreas Protegidas (SNAP). La metodología se basa en la relación beneficio costo para evaluar si es conveniente o no la inversión del Estado en la protección del SNAP. El nivel de inversión del Estado ecuatoriano fue estimado a partir de las necesidades de financiamiento del SNAP. Los beneficios económicos fueron estimados a partir de la oferta de agua de las áreas protegidas que se encuentran a lo largo de la Cordillera de los Andes. Los resultados del estudio demuestran que la relación $\mathrm{B} / \mathrm{C}$ de 1,66 justifica una inversión mayor del Estado en la conservación de sus áreas protegidas.

Palabras Claves: Áreas Protegidas; acceso al agua; microcuencas; inversión; Ecuador

\begin{abstract}
A Commonwealth recently created by Andean province governments of Ecuador seeks to conserve main water sources through ecological corridors. This important agreement set off a review and upgrade of a previous study about the economic value of environmental services provided by protected forest of the National Protected Forest System (SNAP). The method applied was a benefit-cost analysis. The national investment for conservation of the SNAP was the measured of cost and benefits were estimated from water supply originated in 21 national parks and natural reserves of the SNAP located in the Andean Mountains. The upgraded results B/C analysis is 1.66 and validates any State higher investment.
\end{abstract}

Key words: Protected forest, water Access, watersheds, investments, Ecuador. 


\section{INTRODUCCIÓN}

El recurso más importante para garantizar un desarrollo sostenible es sin lugar a dudas el agua. El recurso es tan importante que fue incluido como parte de los objetivos del milenio de las Naciones Unidas (ODM) cuya fecha límite fue el año 2015. El Ecuador declaró que había alcanzado las metas planteadas y con relación al agua la meta fue reducir a la mitad el número de familias sin acceso a agua potable y servicios de saneamiento (Senplades/INEC/PNUD/SNU, 2015). La meta propuesta por las Naciones Unidas en los ODM fue el 66,2 por ciento de la población con acceso a agua potable y servicios de saneamiento, el Ecuador alcanzó el 71,4 por ciento en saneamiento y 77,5 con acceso a agua potable por red pública y que cumple con la Norma INEN 1108, un 18,3\% de agua clorificada (INEC, 2016, 2017). Adicionalmente, se han creado nuevas áreas protegidas para un total de 54 áreas protegidas que forman parte del SNAP en la actualidad, especialmente en regiones marino-costeras con 630 mil hectáreas bajo alguna forma de conservación y manejo sostenible llegando a un total de 33 por ciento del territorio bajo conservación o manejo sostenible (Senplades/INEC/PNUD/SNU, 2015).

Sin embargo y a pesar de loable de los alcances logrados, el objetivo 7, garantizando los derechos de la naturaleza y reduciendo la pérdida de la biodiversidad, aún queda como tarea debido a la limitada inversión que hace el estado ecuatoriano en la conservación de sus áreas naturales protegidas y que forman parte del Sistema Nacional de Áreas Protegidas SNAP. En la proforma del presupuesto general del estado para el año 2018 apenas de 6,7 millones de USD son presupuestadas para la administración y manejo de 54 áreas protegidas del SNAP, pero cubre solo el manejo básico de las áreas protegidas. En el año 2005 un estudio realizado por la empresa Mentefactura en el marco del proyecto MAE/GEF TF 28700 EC estimó las necesidades de financiamiento del SNAP y analizó dos escenarios financieros. El manejo básico, el primer escenario, incluye la implementación de dos programas: (1) administración, control y vigilancia, y (2) planificación participativa con presupuesto estimado de 6,3 millones de USD. El segundo escenario "manejo integral" implica la implementación de una amplia gama de actividades que garantizan el cumplimiento de los objetivos del área protegida en el largo plazo. Este escenario supone la implementación de los dos programas arriba mencionados y tres adicionales: (3) desarrollo comunitario y educación ambiental, (4) turismo y recreación, e (5) investigación, manejo de recursos naturales y monitoreo ambiental con presupuesto estimado de 12,2 millones de USD (MAE, 2005).

En el año 2007, los autores (Rodríguez et al., 2009) participaron en un estudio se realizó como parte de las actividades del proyecto MAE/GEF TF 28700 EC para determinar la valoración económica de los bienes y servicios ambientales del SNAP, los resultados globales del estudio reposan en el Ministerio de Ambiente del Ecuador. Los beneficios fueron estimados a partir de la valoración económica de los servicios ecológicos de las 
áreas protegidas y entre otros bienes y servicios ecológicos, se determinó la oferta de agua y determinó el valor de agua que pagan los usuarios (consumidores) y se estimó un valor de conservación del bosque nativo para garantizar calidad del agua para consumo a partir de varios estudios y programas de pago por servicios ambientales (PSA) realizados en el país.

El estudio tuvo como objetivo principal demostrar el aporte de las áreas protegidas a la economía nacional y a partir de ese valor estimado impulsar en los tomadores de decisiones el diseño de políticas públicas que permitan conservar sus áreas naturales. El valor económico (precio) utilizado para estimar los beneficios económicos del agua fue de $0,05 \mathrm{USD} / \mathrm{m}^{3}$ de agua, un valor promedio de varios estudios de pago por servicios ambientales realizados en el Ecuador (Rodríguez et al., 2009). Este es un valor de conservación de los bosques y vegetación natural donde se encuentran las fuentes de agua, por lo que se considera como el valor (precio) de conservación.

A partir de este valor de conservación, los autores multiplicaron por la cantidad (volumen) de agua concesionado por la Secretaría Nacional del Agua (SENAGUA) hasta la fecha del estudio y solo con relación al agua, los beneficios económicos fueron superiores a 1.515 millones de dólares, sobre la base del valor establecido por la Ley de Agua de 1972. Adicionalmente, los autores determinaron una relación beneficio/costo de 122,39, es decir que por cada dólar invertido en la conservación de las áreas protegidas genera 122,23 dólares de beneficios para el país tomando en cuenta el escenario de financiamiento integral determinado por MAE (2005). Con todo, el estudio se concentró en la oferta del recurso y no analizó la demanda.

El estudio de Rodríguez et al. (2009) retoma importancia debido a que en abril de 2017 11 provincias firmaron un acuerdo de conservación de los páramos y humedales andinos formando una mancomunidad cuyo objetivo principal es cuidar el agua de los páramos a través de corredores ecológicos impidiendo el avance de la frontera agrícola y pastoreo (Moreta et al., 2018).

Debido a la importancia del páramo y sus humedales, como también el hecho que 12 parques nacionales y reservas ecológicas del SNAP se encuentran a lo largo de la región andina del Ecuador y que estas reservas protegidas son las fuentes principales de agua de todas las ciudades y comunidades de los valles interandinos y estribaciones de los Andes Ecuatorianos, el presente estudio tiene como objetivo demostrar los beneficios económicos de las 12 reservas naturales no solo para la mancomunidad, como también para el resto del país. No obstante, difiere del estudio original al concentrarse su análisis desde la demanda y actualizar su tabla de valores con los valores y tarifas establecidos por la nueva Ley de Aguas y su Reglamento. 


\section{UBICACIÓN Y DESCRIPCIÓN GEOGRÁFICA}

El agua es considerada como uno de los recursos más importantes para alcanzar un desarrollo económico y la reducción de la pobreza. La Comisión Mundial del Agua estima que se necesitará doblar la inversión en países del tercer mundo para alcanzar los objetivos del milenio, esto es que se necesitará un incremento anual de aproximadamente 100 billones (millón de millones) de dólares en los próximos 50 años (The International Water Academy, 2003). En el Ecuador la inversión necesariamente deberá realizarse en los páramos y humedales andinos (Figura 1) y apoyar a iniciativas como la de la mancomunidad de los 11 gobiernos provinciales.

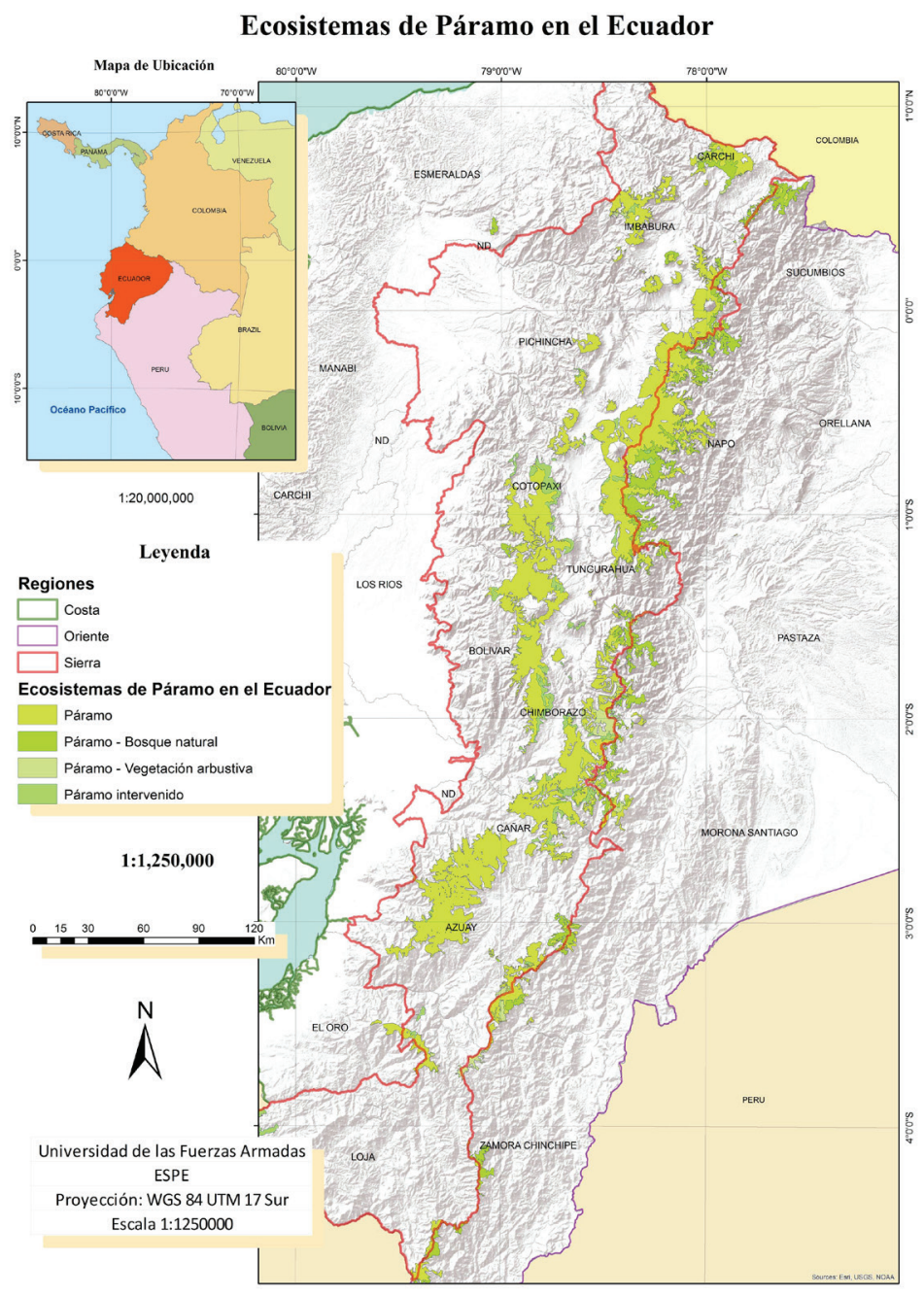

Figura 1. Ubicación del ecosistema de páramo en el Ecuador. 
A nivel nacional parte de las inversiones ya fueron realizadas mucho antes de la declaración de los ODM a través de la creación áreas protegidas y entre los objetivos de su creación está el conservar el recurso agua. Diecisiete de las 54 áreas protegidas del Ecuador se encuentran en la Cordillera de los Andes (Tabla 1) donde el ciclo del agua empieza (captura, filtración, y almacenaje). De estas 17 áreas, solo 12 fueron tomadas en cuenta en el análisis. Hay 5 reservas que fueron creadas posteriormente a la estimación de la oferta de agua que nace en las reservas naturales y del cálculo de la demanda a partir de los datos de Senagua. Por otro lado, es necesario aclarar que la vegetación introducida ya sea como bosques y pastos plantados no fueron incluidos como parte de la vegetación natural y es así que el Parque Nacional Cotopaxi, por ejemplo, tiene solo 71 por ciento de vegetación andina y montana a pesar de que se encuentra completamente entre las cordillera Real y Occidental de los Andes.

Tabla 1. Áreas Protegidas del Ecuador en la Zona Andina

\begin{tabular}{lccc}
\multicolumn{1}{c}{ ÁREA PROTEGIDA } & Año creación & $\begin{array}{c}\text { Área total } \\
\text { (ha) }\end{array}$ & $\begin{array}{c}\text { \% Vegetación } \\
\text { andina y montana }\end{array}$ \\
\hline PARQUE NACIONAL CAJAS & 1977 & 28.808 & 96,50 \\
\hline RESERVA ECOLOGICA CAYAMBE-COCA & 1970 & 402.000 & 75,69 \\
\hline PARQUE NACIONAL COTOPAXI & 1975 & 33.393 & 71,03 \\
\hline PARQUE NACIONAL LLANGANATES & 1996 & 219.707 & 91,71 \\
\hline PARQUE NACIONAL PODOCARPUS & 1982 & 146.280 & 93,73 \\
\hline PARQUE NACIONAL SANGAY & 1979 & 517.725 & 77,62 \\
\hline RESERVA ECOLOGICA ANTISANA & 1993 & 120.000 & 68,15 \\
\hline RESERVA ECOLOLOGICA EL ANGEL & 1992 & 15.715 & 96,44 \\
\hline RESERVA ECOLOGICA COTACACHI- & 1968 & 243.638 & 48,20 \\
\hline CAYAPAS & 1996 & 149.900 & 90,12 \\
\hline RESERVA ECOLOGICA LOS ILINIZAS & 1978 & 3.383 & 96,03 \\
\hline RESERVA GEOBOTANICA PULULAHUA & 1987 & 58.560 & 62,73 \\
\hline RESERVA FAUNISTICA CHIMBORAZO & 1982 & 500 & 100,00 \\
\hline REFUGIO DE VIDA SILVESTRE PASOCHOA & 1979 & 227 & 39,07 \\
\hline ÁREA NACIONAL DE RECREACION EL & & $1 ’ 939.609$ & 47,49 \\
\hline BOLICHE & & & \\
\hline TOTAL & & & \\
\hline
\end{tabular}

Fuente: MAE (2005); Elaboración: Autores.

Los parques nacionales, reservas ecológicas, refugios del SNAP ubicados a lo largo de la Cordillera de los Andes tiene una gran variedad de ecosistemas debido a que se encuentran entre varios pisos altitudinales desde el bosque húmedo tropical, pasando por bosque nublado y de neblina, bosque andino hasta los páramos de pajonal y almohadillas (Figura 2). Esta variedad permite a estas reservas naturales tener una enorme biodiversidad con un alto número de especies endémicas. Una breve descripción de las 12 áreas naturales objetivos de este estudio del SNAP se presenta a continuación (MAE, 2018). 


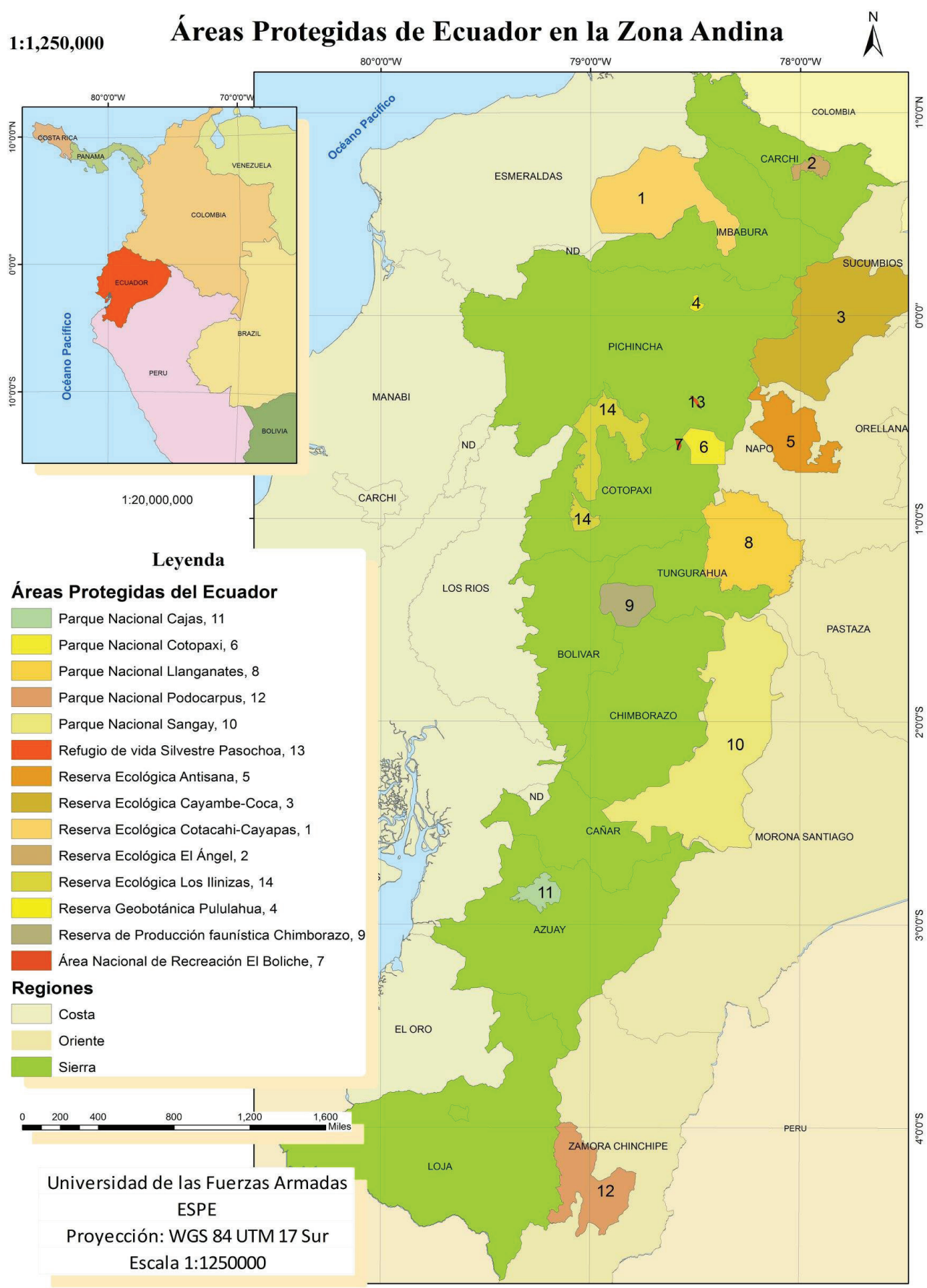

Fuente: MAE, 2015. Elaboración: Autores

Figura 1. Áreas protegidas del SNAP en la zona andina 
El Parque Nacional Cajas tiene una superficie de 28.808 hectáreas y fue creado el 04/071977 Registro Oficial A203 y publicado en el R. O. No. 317 del 4 de julio de 1977. Está ubicada entre los 3.200 y 4.450 metros de altura. La temperatura promedio de la zona está en los 10 grados centígrados y las precipitaciones van desde los 1.200 a 2.000 $\mathrm{mm}$ anuales, características pertenecientes al ecosistema de bosque alto andino y páramo en el Ecuador.

El Parque Nacional Cayambe Coca fue creado por el gobierno del Ecuador mediante Decreto Ejecutivo No. 818 del 17 de noviembre de 1970 y publicado en el R. O. No. 104 del 20 de noviembre de 1970. La RECAY tienen 403.103 hectáreas de superficie, localizadas en la zona integrada por las provincias norandinas de Imbabura y Pichincha y las nororientales de Napo y Sucumbíos, a una altitud que varía desde los 750 hasta los 5.790 metros sobre el nivel del mar, clima frío, templado y subtropical según la altura, con precipitaciones anuales de 500 a 3.000 milímetros. El parque nacional tiene una gran variedad de ecosistemas desde páramos y humedales de páramo hasta bosque nublado tropical.

El Parque Nacional Cotopaxi se encuentra ubicado en las provincia de Pichincha, Cotopaxi, y Napo, posee una extensión de 33393 has que incluyen varias estructuras montañosas como el volcán Cotopaxi, el Rumiñahui, Sincholagua; la laguna de Limpiopungo y algunas ruinas arqueológicas. Fue creado el 11 de agosto de 1975 mediante Acuerdo Ministerial A-259 y publicado en R. O. No. 876 del 27 de agosto de 1975. Posee un clima frío de altura cuyas temperaturas por la mañana pueden alcanzar los 18 a 20 grados y en las tardes y noches descender a los 3 o 4 grados. PNC se encuentra entre los $3000 \mathrm{~m}$ llegando hasta los $5897 \mathrm{msnm}$. El ecosistema predominante es de páramo principalmente de pajonal y arbustivo andino.

El Parque Nacional Llanganates fue creado el 18 de enero de 1996 por Resolución No. 00002 y publicado en el R. O. No. 907 del 19 de marzo de 1996. El parque nacional tiene un área de 219.707 Has., este territorio protegido ocupa además las provincias de Cotopaxi, Napo y Pastaza. Se trata principalmente de un bosque andino y páramo incluyendo de almohadillas, tiene a los macizos montañosos de los Llanganates como principal atractivo. Forman parte de estos el denominado Cerro Hermoso, el sistema lacustre Pisayambo y los torrentes de las estribaciones montañosas. También hay bosques amazónicos exuberantes.

El Parque Nacional Podocarpus se estableció mediante Acuerdo Ministerial No. 398 del 15 de diciembre de 1982 y publicado en el R. O. No. 404. Tiene una extensión de 146.280 hectáreas. El parque nacional se ubica en la zona de confluencia entre los húmedos Andes del norte y los bosques más secos de la zona tumbesina del sur.

El Parque Nacional Sangay se estableció mediante acuerdo ministerial No. 190 del 26 de julio de 1979 y publicado en el R. O. No. 840. Tiene una superficie de 271.925, hectáreas que ocupan gran parte de las jurisdicciones territoriales de las provincias 
de Tungurahua, Chimborazo y Morona Santiago. En 1983, la UNESCO declara al Parque Nacional Sangay Patrimonio Mundial de la Humanidad, reconociendo su gran importancia biológica, ecológica y cultural que abarca múltiples ecosistemas existentes desde la región amazónica ecuatoriana hasta el páramo húmedo de almohadillas y las nieves perpetuas. El 20 de mayo de 1992, mediante registro Oficial $\mathrm{N}^{\circ}$. 939, se ampliaron los límites a 517.725 hectáreas, en donde se incluye parte de la Provincia del Cañar.

La Reserva Ecológica Antisana fue creada por Resolución No. 18 del 21 de julio de 1993 y publicado en R. O. No. 265 del 31 de agosto de 1993. Está ubicada entre las provincias de Pichincha y Napo, tiene una superficie de 120.000 hectáreas y está situada entre los 1.400 y 5.700 metros sobre el nivel del mar. Lleva el nombre del volcán Antisana cubierto de nieves perpetuas y bosques andinos, lagunas y ríos en sus alrededores y, en el sector oriental, vegetación en la llanura amazónica que también la integra.

La Reserva Ecológica El Ángel se creó el 5 de agosto de 1992 por Decreto Ejecutivo A-415 y publicado en el R. O. No. 21 del ocho de septiembre de 1992. La Reserva esta ubicada en la jurisdicción del cantón Espejo, provincia del Carchi, con una extensión de 15.715 hectáreas y una altura que va desde los 3.644 hasta los 4.768 metros sobre el nivel del mar, con un clima frío de páramo, temperaturas que varían entre 0 y 18 grados centígrados y precipitaciones anuales de 1.000 a 1.500 milímetros.

La Reserva Ecológica Cotacachi Cayapas fue creada el 29 de agosto de 1968 con Decreto Ejecutivo No. 1468, publicado en el R.O. No.17 del 20 de septiembre de 1968. Se encuentra localizada entre las provincias de Esmeraldas e Imbabura, con una extensión de 243.638 hectáreas y altitudes que ascienden desde uno hasta los 1.600 metros sobre el nivel del mar, en la zona baja, que registra una temperatura promedio de 25 grados centígrados; y de 1.600 a 4.939 metros en la zona alta, con una temperatura de 15 grados centígrados. El clima, determinado por la topografía, vana de templado periódicamente húmedo a frío de alto andino y de tropical lluvioso a templado periódicamente seco. Con relación a las precipitaciones anuales, éstas varían entre 3.000 a 5.000 milímetros.

La Reserva Ecológica Illinizas fue creada el dos de diciembre de 1996 mediante Resolución No. 066 y el decreto ejecutivo de creación fue publicado en el R. O. No. 92 del 12 de diciembre de 1996, con una superficie de 149.900 hectáreas, localizadas entre los 800 y 5.265 metros sobre el nivel del mar. La temperatura es de 0 grados centígrados en la zona alta y alcanza un máximo de 24 grados en la zona baja, con precipitaciones de entre 1.000 y 2.000 milímetros. La reserva se ubica en ecosistemas de bosque nublado, bosque andino y arbustivo, y páramo de pajonal.

La Reserva Geobotánica Pululahua fue creada por decreto supremo No. 194 y Acuerdo Ministerial No. 137 del 28 de enero de 1966 y publicado en el R. O. No. 715 del 21 de marzo de 1978, está ubicada al noroccidente de San Antonio de Pichincha, a 23 kilómetros de la ciudad de Quito, limitada al norte por el río Guayllabamba; al sur, las 
poblaciones de Calacalí y Caspigasi del Carmen; al este, el cerro Sincholagua, la loma El Lavadero y la quebrada Aguacatal; y al oeste, el río Blanco y la quebrada de Playa. Tiene una gran variedad de microclimas donde se destaca el bosque andino, arbustos y bosque de neblina.

La Reserva Faunística Chimborazo fue creada el 26 de octubre de 1987, mediante Acuerdo Interministerial No. 437 y el acuerdo fue publicado en RT. O. No. 806 del 9 de noviembre de 1987, cubre una superficie de 58.560 hectáreas localizadas entre las provincias de Chimborazo, Tungurahua y Bolívar, con altitudes que van desde los 3.800 hasta los 6.310 metros sobre el nivel del mar. El clima predominante es el frío de alto andino con variaciones de templado permanente húmedo a templado periódicamente seco, con temperaturas de entre 0 y 10 grados centígrados.

El Refugio de Vida Silvestre Pasochoa fue creado mediante acuerdo ministerial del 26 de noviembre de 1982, por las características especiales de la zona, declaró Bosque y Vegetación Protectora a una superficie de 500 hectáreas de la Hacienda "Pilopata", propiedad del Ministerio de Salud. En 1996 y dada la importancia del área, el Pasochoa fue declarado Refugio de Vida Silvestre, con una extensión de 502 hectáreas de bosque andino.

El Área Nacional de Recreación El Boliche fue creada el 26 de julio de 1979 mediante acuerdo ministerial A-322 publicado en el R. O. No. 69 del 20 de noviembre de 1979, está ubicada a 60 kilómetros de la ciudad de Quito y a 30 kilómetros de Latacunga en los límites de las provincias de Cotopaxi y Pichincha; tiene una superficie total de 227 hectáreas y la elevación más alta de la zona es el cerro Sunfana de 3.704 metros de altura. El rango altitudinal está comprendido entre los 3.000 y 3.600 metros sobre el nivel del mar.

\section{METODOLOGÍA}

La creación de las áreas protegidas es en sí un paso importante para garantizar el acceso a bienes y servicios ambientales, mejorar el bienestar de las personas y reducir la pobreza. Sin embargo, es necesario determinar la eficiencia económica de mantener estas áreas protegidas para lo cual se requiere establecer tanto los beneficios definidos como.

$$
B=P \times Q
$$

La estimación de la oferta de agua de las áreas protegidas fue estimada a partir de las estaciones de meteorológicas cercanas a cada área protegida (Tabla 2) y estimó los valores de escorrentía, evaporación e infiltración. El modelo general de escorrentía es el siguiente:

$$
Q+G=P-E-I-E T
$$


Donde, Q es caudal superficial de la cuenca a ser analizada, G es el flujo neto de aguas subterráneas desde la cuenca hacia el exterior, P es la precipitación, E es la evaporación, I es la infiltración en el suelo, y ET es la evapotranspiración la cual es puede ser un valor insignificante por lo que no fue tomado en cuenta.

Tabla 2. Estaciones meteorológicas cercanas a las áreas protegidas

\begin{tabular}{|c|c|c|c|c|}
\hline ÁREA PROTEGIDA & NOMBRE & COD. & $\begin{array}{l}\text { ALTURA } \\
(\mathrm{m})\end{array}$ & $\begin{array}{c}\text { RÍO DE } \\
\text { MEDICIÓN }\end{array}$ \\
\hline $\begin{array}{l}\text { PARQUE NACIONAL } \\
\text { CAJAS }\end{array}$ & $\begin{array}{l}\text { MATADERO EN } \\
\text { SAYAUSI }\end{array}$ & H-896 & 2645 & $\mathrm{~S} / \mathrm{N}$ \\
\hline $\begin{array}{l}\text { PARQUE NACIONAL } \\
\text { COTOPAXI }\end{array}$ & PITA AJ SALTO & H-158 & 3550 & RIO PITA \\
\hline $\begin{array}{l}\text { PARQUE NACIONAL } \\
\text { LLANGANATES }\end{array}$ & $\begin{array}{l}\text { JATUNYACU DJ } \\
\text { ILOCULIN }\end{array}$ & H-721 & 570 & RIO ILOCULIN \\
\hline $\begin{array}{l}\text { PARQUE NACIONAL } \\
\text { PODOCARPUS }\end{array}$ & $\begin{array}{l}\text { UCHIMA AJ } \\
\text { CHAMBA }\end{array}$ & H-627 & 1544 & $\mathrm{~S} / \mathrm{N}$ \\
\hline $\begin{array}{l}\text { PARQUE NACIONAL } \\
\text { SANGAY }\end{array}$ & $\begin{array}{l}\text { OZOGOCHE EN } \\
\text { LOS LAGOS }\end{array}$ & H-783 & 3715 & $\mathrm{~S} / \mathrm{N}$ \\
\hline $\begin{array}{l}\text { RESERVA ECOLOGICA } \\
\text { ANTISANA }\end{array}$ & $\begin{array}{l}\text { MISAHUALLI EN } \\
\text { COTUNDO }\end{array}$ & 20 & 800 & RIO M \\
\hline \multirow[t]{2}{*}{$\begin{array}{c}\text { RESERVA } \\
\text { ECOLOLOGICA EL } \\
\text { ANGEL }\end{array}$} & IA & 91 & 20 & JATIVA \\
\hline & $\begin{array}{l}\text { BOBO EN PLANTA } \\
\text { ELEC. TULCÁN }\end{array}$ & H-093 & 2880 & $\mathrm{~S} / \mathrm{N}$ \\
\hline $\begin{array}{l}\text { RESERVA ECOLOGICA } \\
\text { CAYAMBE-COCA }\end{array}$ & $\begin{array}{l}\text { GUAYLLABAMBA } \\
\text { DJ PISQUE }\end{array}$ & -148 & 1690 & $\begin{array}{c}\text { RÍO } \\
\text { GUAYLLABAMBA }\end{array}$ \\
\hline $\begin{array}{l}\text { RESERVA ECOLOGICA } \\
\text { COTACACHI-CAYAPAS }\end{array}$ & MIRA EN LITA & H-011 & 475 & RIO LITA \\
\hline $\begin{array}{l}\text { RESERVA ECOLOGICA } \\
\text { LOS ILINIZAS }\end{array}$ & $\begin{array}{l}\text { TOA } \\
\text { PIL }\end{array}$ & H-161 & 820 & RIO TOACHI \\
\hline $\begin{array}{c}\text { RESERVA } \\
\text { GEOBOTANICA } \\
\text { PULULAHUA } \\
\end{array}$ & $\begin{array}{l}\text { GUAYLLABAMBA } \\
\text { DJ PACHIJAL }\end{array}$ & H-147 & 410 & $\begin{array}{c}\text { RIO } \\
\text { GUAYLLABAMBA }\end{array}$ \\
\hline $\begin{array}{l}\text { RESERVA FAUNISTICA } \\
\text { CHIMBORAZO }\end{array}$ & $\begin{array}{l}\text { BALSACON EN } \\
\text { SAN ANDRES }\end{array}$ & 91 & 3000 & $\cdots$ \\
\hline $\begin{array}{c}\text { REFUGIO DE VIDA } \\
\text { SILVESTRE PASOCHOA }\end{array}$ & $\begin{array}{l}\text { SAN PEDRO EN } \\
\text { MACHACHI }\end{array}$ & H-159 & 2680 & $\cdots$ \\
\hline $\begin{array}{c}\text { AREA NACIONAL } \\
\text { DE RECREACION EL } \\
\text { BOLICHE }\end{array}$ & PITA AJ SALTO & H-158 & 3550 & RIO PITA \\
\hline
\end{tabular}

Los costos fueron determinados a partir del presupuesto estimado para la conservación de las áreas protegidas del estudio fue realizado por la empresa Mentefactura antes mencionado. 
Por otro lado, los beneficios fueron estimados a partir de un estudio realizado por Hexagon Consultores sobre valoración económica de los servicios ecológicos de las áreas protegidas y la elaboración de la estrategia de financiamiento del SNAP. En este estudio se estimó, entre otros bienes y servicios ecológicos, la cantidad de agua que es ofertada por las áreas protegidas, se determinó el valor de agua que pagan los usuarios (consumidores) y se estimó un valor de conservación del bosque nativo para garantizar calidad del agua para consumo a partir de varios estudios y programas de pago por servicios ambientales (PSA) realizados en el país.

Para determinar si la conservación de áreas protegidas es conveniente para el país se determinó la tasa costo beneficio la cual se sustenta en el principio de obtener y alcanzar niveles considerables de producción con el mínimo uso de recursos, que en términos de conservación de áreas protegidas, simplemente dice alcanzar los máximos beneficios a un mínimo costo.

$$
\frac{B}{C}=\frac{\sum_{t=o}^{T} \frac{b_{t}}{(1+r)^{t}}}{\sum_{t=o}^{T} \frac{c_{t}}{(1+r)^{t}}}
$$

Donde $T$ es el tiempo del análisis, $b_{t}$ corresponde a los beneficios, $c_{t}$ son los costos totales, y $r$ es la tasa de descuento

Como referencia, el criterio para cualquier proyecto donde la tasa $\mathrm{B} / \mathrm{C}^{3} 1.0$ es considerada como aceptable. Si la tasa es igual a 1, el proyecto produciría cero beneficios netos sobre la vida útil del proyecto. Si la tasa es menos que 1 significaría que le proyecto está produciendo pérdidas desde el punto de vista económico (Randall, 1987).

\section{RESULTADOS}

La estimación del aporte de agua de las áreas protegidas andinas al país se resume en la Tabla 3. El volumen total de agua que nace en las áreas protegidas estimado es de 548 mil millones de metros cúbicos al año, la demanda por otro lado es de 68 mil millones de metros cúbicos al año. La gran mayoría de la demanda es para el riego, un resultado esperado, aproximadamente el $81 \%$ de la demanda de agua es para sistemas de riego de acuerdo con la FAO y su sistema AQUASTAT (FAO/AQUASTAT, 2015).

El promedio del aporte (oferta) de las áreas protegidas es aproximadamente de 39 mil millones de $\mathrm{m} 3$ de agua para distintos usos (Tabla 3). Las áreas naturales que más aportan son el Parque Nacional Llanganates y la Reserve Ecológica Cotacachi Cayapas que aportan con el $69 \%$ de la oferta de agua. Sin embargo, si se considera la demanda, el 
Parque Nacional Cayambe Coca es el más importante con relación a sus usos tanto en el riego, consumo e industrial. La demanda de este parque nacional representa el $12,1 \%$ de la oferta total de agua.

Tabla 3. Oferta de agua de las áreas protegidas andinas y demanda por actividad

\begin{tabular}{|c|c|c|c|c|c|}
\hline ÁREA PROTEGIDA & $\begin{array}{l}\text { Vol l m e n } \\
\text { anual }-\mathbf{m}^{3} / \mathbf{a n ̃ o -} \\
\text { (miles) }\end{array}$ & $\begin{array}{l}\text { D e m a n d a } \\
- \text { m }^{3} / \text { a ñ o- } \\
\text { (miles) }\end{array}$ & $\begin{array}{l}\text { Riego }-\mathbf{m}^{3} / \\
\text { año- (miles) }\end{array}$ & $\begin{array}{l}\text { Cons u mo } \\
- \text { m }^{3} / \mathbf{a} \tilde{n} 0- \\
\text { (miles) }\end{array}$ & $\begin{array}{l}\text { Industrial } \\
-\mathbf{m}^{3} / \text { a ñ } 0- \\
\text { (miles) }\end{array}$ \\
\hline $\begin{array}{l}\text { PARQUE NACIONAL } \\
\text { CAJAS }\end{array}$ & $1.185 .378,66$ & $600.188,74$ & $486.152,88$ & $78.024,54$ & $36.011,32$ \\
\hline $\begin{array}{l}\text { PARQUE NACIONAL } \\
\text { COTOPAXI }\end{array}$ & $1.872 .340,00$ & $6.463,05$ & $5.235,07$ & 840,20 & 387,78 \\
\hline 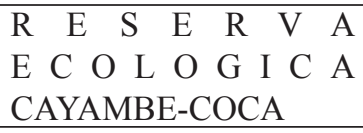 & $17.846 .426,37$ & $66.242 .837,58$ & $53.656 .698,44$ & $8.611 .568,89$ & $3.974 .570,25$ \\
\hline $\begin{array}{l}\text { PARQUE NACIONAL } \\
\text { LLANGANATES }\end{array}$ & $174.539 .361,70$ & $997.941,74$ & $808.332,81$ & $129.732,43$ & $59.876,50$ \\
\hline $\begin{array}{l}\text { PARQUE NACIONAL } \\
\text { PODOCARPUS }\end{array}$ & $9.738 .411,22$ & $1.229,90$ & 996,22 & 159,89 & 73,79 \\
\hline $\begin{array}{l}\text { PARQUE NACIONAL } \\
\text { SANGAY }\end{array}$ & $47.448 .163,49$ & $88.916,70$ & $72.022,53$ & $11.559,17$ & $5.335,00$ \\
\hline 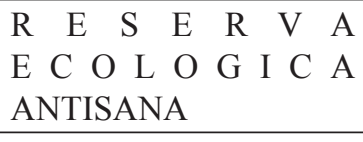 & 68.12 & $3.027,46$ & $2.452,24$ & 393,57 & 181,65 \\
\hline 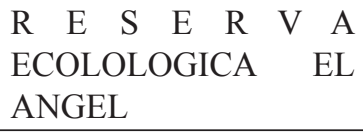 & 2.240 . & $40.744,51$ & $33.003,05$ & $5.296,79$ & $2.444,67$ \\
\hline 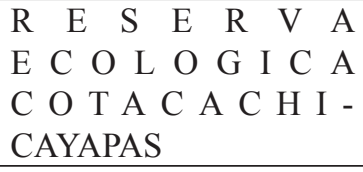 & $203.688 .240,01$ & $184.855,20$ & $149.732,71$ & $24.031,18$ & $11.091,31$ \\
\hline $\begin{array}{lllll}\mathrm{R} \quad \mathrm{E} \quad \mathrm{S} & \mathrm{E} & \mathrm{R} & \mathrm{V} & \mathrm{A} \\
\text { ECOLOGICA } & & \text { LOS } \\
\text { ILINIZAS } & & & \\
\text { lLINAS }\end{array}$ & $17.455 .593,20$ & $44.449,27$ & $36.003,91$ & $5.778,40$ & $2.666,96$ \\
\hline $\begin{array}{l}\mathrm{R} \quad \mathrm{E} \quad \mathrm{S} \quad \mathrm{E} \quad \mathrm{R} \quad \mathrm{V} \text { A } \\
\text { G E O B O T A N I C A } \\
\text { PULULAHUA }\end{array}$ & $738.320,75$ & 283,82 & 229,90 & 36,90 & 17,03 \\
\hline $\begin{array}{l}R \quad E \quad S \quad E \quad R \quad V \quad A \\
\text { F A U N I S T I C A } \\
\text { CHIMBORAZO }\end{array}$ & $1.269 .544,92$ & $83.696,54$ & $67.794,20$ & $10.880,55$ & $5.021,79$ \\
\hline $\begin{array}{l}\text { REFUGIO DE } \\
\text { VIDA SILVESTRE } \\
\text { PASOCHOA }\end{array}$ & 2,36 & $.040,69$ & 842,96 & 135,29 & 62,44 \\
\hline $\begin{array}{l}\text { AREA NACIONAL } \\
\text { DE RECREACION EL } \\
\text { BOLICHE }\end{array}$ & $1.872 .340,00$ & 0,00 & 0,00 & 0,00 & 0,00 \\
\hline TOTAL & $548.050 .046,91$ & $68.295 .675,20$ & $55.319 .496,91$ & $8.878 .437,78$ & $4.097 .740,51$ \\
\hline
\end{tabular}


Una vez establecida la cantidad, el volumen de agua tanto de la oferta como de la demanda, la estimación de los beneficios se realizaron sobre la base de 3 principales uso y los valores (precios) que Senagua tiene establecido para cada actividad (Tabla 4).

Tabla 4: Tarifas y valores para los usos del agua

\begin{tabular}{lr}
\hline TIPO DE USO & \multicolumn{1}{c}{ VALOR } \\
\hline Valor de conservación (US\$/m3 & 0,0012806 \\
Valor consumo Senagua (US\$/m3 & 0,00039 \\
Valor riego Senagua (promedio) (US\$/m3) & 0,0000875 \\
Valor electricidad Senagua (US\$/m3) & 0,0049 \\
Valor electricidad industrial (US\$m) & 0,001 \\
\hline
\end{tabular}

Fuente: SENAGUA, 2017; Elaboración: Autores

Los beneficios estimados de las 12 áreas protegidas del SNAP alcanzan un valor de 20’307.529,31 USD/año basados en las tarifas establecidas por Senagua (Tabla 5). El resultado de los beneficios económicos del bien y servicio ambiental agua es un flujo, es decir es un valor anual. El mayor aporte a este valor proviene del agua para consumo humano para el cual se aplicó el valor de conservación, es decir el valor de la disposición a pagar para la conservación de los ecosistemas donde se encuentran las principales fuentes de agua de las ciudades. El beneficio económico tanto de riego como industrial son muy parecidos y se debe a las distintas tarifas establecidas por Senagua; sin embargo hay que tomar en cuenta que la diferencia en la demanda es enorme.

Tabla 5. Beneficios económicos por el bien y servicio ambiental agua

\begin{tabular}{lcc}
\hline \multicolumn{1}{c}{ USO } & m3/año (miles) & \multicolumn{1}{c}{ Beneficio } \\
\hline Demanda & $68.295 .675,20$ & - \\
Riego & $55.319 .496,91$ & $4.840 .455,98$ \\
Consumo & $8.878 .437,78$ & $11.369 .332,82$ \\
Industrial & $4.097 .740,51$ & $4.097 .740,51$ \\
\hline Total & & $20.307 .529,31$ \\
\hline
\end{tabular}

Fuente SENAGUA, 2006; FAO AQUASTAT, 2005; Elaboración: Autores

En análisis beneficio costo para determinar si la inversión en las áreas protegidas es rentable o no, se estimó el valor requerido para financiar el SNAP. El nivel de financiación integral del SNAP alcanza un valor de 12’211.681,00 dólares al año en el marco de los dos escenarios de financiamiento del estudio del Ministerio del Ambiente (2005). Los resultados (Tabla 6) demuestran que los beneficios de protección de las 12 áreas protegidas sobrepasan los costos del financiamiento de todas las 34 áreas protegidas existentes en el año 2017. 
Tabla 6 Análisis beneficio-costo $(\mathrm{B} / \mathrm{C})$

\begin{tabular}{lrr}
\hline & \multicolumn{1}{c}{ B ENEF ICIOS } & \multicolumn{1}{c}{ B/C } \\
\hline Á r e a s & 20,31 & 1,66 \\
Protegidas & 34.853 & 0,00058 \\
PGE 2017 & $103.100,00$ & 0,0002 \\
PIB 2017 & $1.302,00$ & 0,016 \\
\hline
\end{tabular}

Fuente: BCE: 2018; Elaboración: Autores

La relación beneficio costo es de 1,66 que establece que por cada dólar invertido se recupera 66 centavos de ganancia neta. Los beneficios totales solo por el bien y servicio ambiental agua comparados con el presupuesto general del estado (PGE) es mínimo, de igual forma ocurre con relación al PIB. Sin embargo, se debe considerar que solo se habla de un bien y servicio ambiental. Si se añadiera otros servicios ambientales como la función de sumidero y almacenamiento de carbono, estos valores cambiarían radicalmente.

Der igual forma, si se compara los beneficios de protección de las áreas protegidas con el subsidio a la gasolina, la cual beneficia en su mayoría a quienes si pueden pagar un precio real, la protección de las áreas protegidas tiene más sentido y genera directos beneficios para el bienestar de la población.

\section{CONCLUSIONES}

Los resultados presentados en la Tabla 6 no dejan duda que la inversión en el financiamiento de todo el sistema de SNAP es provechoso para el país. El volumen de agua ofertado por solo las 12 áreas protegidas de zonas andinas y montanas es suficiente justificativo para que el Gobierno Nacional invierta más en la protección de todo el SNAP, actualmente solo cubre parte (escenario básico de financiamiento) y tomando en cuenta que estos escenarios fueron estimados cuando el número de áreas protegidas eran 34, hoy en día son 52 áreas protegidas, 18 nuevas las cuales requieren un financiamiento global para su real conservación.

Los valores presentados en el presente estudio difieren de aquellos presentados por Rodríguez et al. (2009) debido a que se actualizaron los valores, sobre todo tarifas establecidas en la nueva Ley de Aguas, el valor de conservación. En el estudio original, el valor de conservación se multiplicó directamente por la oferta de agua que nace de las áreas protegidas. En este estudio, se concentra principalmente en la demanda y en los distintos tipos de uso que se muestran en la Tabla 6. Adicionalmente, el PIB en 2007 llegó a 44 mil millones, hoy sobrepasa los 100 mil millones.

Por otro lado, las tarifas utilizadas en el presente estudio son más bajas en comparación con las existentes en 2007, una indicación que no se cubre los costos totales que incluyen costos ambientales y sociales. 
En definitiva, el presente estudio representa un apoyo a la iniciativa de la mancomunidad de 11 provincias que firmaron un acuerdo de conservación de los páramos y humedales andinos formando con el objetivo principal es cuidar el agua de los páramos a través de corredores ecológicos impidiendo el avance de la frontera agrícola y pastoreo.

\section{REFERENCIAS}

INEC. (2016). Ecuador en Cifras: Medición de los indicadores ODS de Agua, Saneamiento e Higiene (ASH) en el Ecuador. Instituto Nacional de Estadísticas y Censos, Quito, Ecuador. $25 \mathrm{pp}$.

INEC. (2017). Ecuador en Cifras: Estadística de Información Ambiental Económica en Gobiernos Autónomos Descentralizados Municipales Agua y Alcantarillado (2015). Instituto Nacional de Estadísticas y Censos, Quito, Ecuador. 23 pp.

Ministerio del Ambiente. (2018). Punto Verde, Sistema Nacional de Áreas Protegidas, Quito, Ecuador. Recuperado: http://areasprotegidas.ambiente.gob.ec/es/todas-areas-protegidaspor-region?t=S

Ministerio del Ambiente. (2005). Análisis de las Necesidades de Financiamiento del Sistema Nacional de Áreas Protegidas del Ecuador. Génesis Ediciones, Quito, Ecuador.

Moreta, M., Castillo, L., y Benalcázar, W. (2018). 11 provincias del Ecuador se integran a favor del páramo. El Comercio, Actualidad, Ecuador. Compañía Anónima El Comercio, 10 de abril de 2018. Recuperado en: https://www.elcomercio.com/actualidad/ecuadorprovincias-agua-paramo-humedales.html

Randall, A. (1987) Resource Economics: An Economic Approach to Natural Resource and Environmental Policy. John Wiley \& Son, Inc. Publishers. New York. pp. 247-250.

Rodríguez, F., Galárraga, R., Salazar, R., y Narváez, N. (2009). Ecuador y los objetivos del Milenio: inversiones para la conservación del agua. Letras Verdes, Vol. 3, pp. 23-25.

Senagua. 2017. Reforma Acuerdo Ministerial No. 2017-1522. Secretaría Nacional del Agua, Gobierno de la República del Ecuador, 27 de junio de 2017. Quito, Ecuador.

Senplades/INEC/PNUD/SNU. (2015). Objetivos del Milenio: Balance Ecuador 2014.Secretaría Nacional de Planificación y Desarrollo, Secretaría de Seguimiento y Evaluación. Senplades - INEC - PNUD - SNU / $1^{\circ}$ Edición, Quito, Ecuador. 32pp.

The International Water Academy (2003) Water for the Poorest: Responding to the Millennium Goals. A Conference in Support of the United Nations Commission for Sustainable Development, 4-5 November 2003. 